\title{
INFLUENCIA DEL pH SOBRE LAS ALGAS EDAFICAS
}

\author{
M. HERNANDEZ MARINE, A. NOGUEROL SEOANE Y \\ J. SEOANE CAMBA *
}

\section{RESUMEN:}

Se ponen de manifiesto las relaciones entre el $\mathrm{pH}$ del suelo y la presencia dealgas, en un total de 40 muestras. Las algas pertenecen a 60 géneros de las divisiones Cyanophyta, Bacillariophyta y Chlorophyta que han sido aisladas usando la técnica de cultivos de enriquecimiento.

También se relacionan estos grupos algales con el porcentaje de materia orgánica presente en el substrato.

\section{SUMMARY:}

We clear-up the relationship between the soil $\mathrm{pH}$ and the algal presence, on atotal of 40 samples. The algae belong to 60 different genera of the classes Cyanophyta, Bacillariophyta and Chlorophyta which have been isolated using the enrichment culture technique.

We also relate these algae groups wits the percentage of organic matter present in the substratum.

Las algas edáficas se encuentran en todos los tipos de suelos y según los estudios efectuados en los últimos años (Shtina, 1960; Pandey, 1965; Akiyama, 173; Halperín, 1976), actúan como un componente importante, formando materia orgánica, fijando nitrógeno y reteniendo humedad.

Estamos realizando trabajos sobre las algas del suelo de la región catalana tanto sistemáticos como ecológicos, por el método de cultivos de enriquecimiento y hemos querido estudiar los factores que influían sobre su heterogeneidad.

La riqueza en algas de un suelo determinado depende de un gran númerode factores físicos y químicos como son: luz, temperatura, humedad, nutrientes, etc. Nosotros nos hemos centrado en dos de ellos, el pH y la materia orgánica. 
El pH por su facilidad de medida y por la innegable influencia que ejerce sobre las algas del suelo, ha sido uno de los factores más estudiados y que cuentan con más amplia bibliografía (Lund, 1947; Shtina, 1959; Brock, 1973).

La mayor parte de las especies crecen sobre suelos neutros o ligeramente alcalinos, siendo entre 5,5 y 8,5 donde se encuentran en mayor número.

En suelos fuertemente ácidos predominan las clorofíceas y no se encuentran cianofíceas por debajo de $\mathrm{pH}$ 4'5, éstas dominan en suelos alcalinos, incluyendo en este grupo los salinos y los áridos que suelen ser también básicos en casi todo el mundo.

Para este estudio trabajamos con un total de 40 muestras, cuyos márgenes de $\mathrm{pH}$ se encuentran comprendidos entre 4,9 y 8,05 .

Desde un principio separamos los suelos en dos categorías, los que tenían un $\mathrm{pH}$ por debajo de 7 y los que se encontraban por encima de esta cifra.

Los resultados nos han confirmado lo que ya sabíamos por la bibliografía a pHs muy ácidos no se encuentran prácticamente cianofíceas.

La mayor heterogeneidad algal se encuentra a $\mathrm{pHs}$ alcalinos, pero la reciprocidad no es cierta, no es seguro que en un suelo de $\mathrm{pH}$ alcalino, se encuentren muchos táxones distintos.

Un pequeño número de diatomeas nos ha aparecido en casi todas las muestras. Todas ellas son especies cosmopolitas y se encuentran independientemente de la variación de los valores de $\mathrm{pH}$.

Para comprobar estadísticamente la relación entre el pH y la heterogeneidad algal, hemos realizado las correspondientes gráficas. En la figura número $1 \mathrm{~A}$, hemos colocado en abscisas el $\mathrm{pH}$ y en ordenadas el número total de especies algales. Hemos hecho lo mismo con las cianofíceas (fig. 1 B) y con las clorofíceas (fig. 1 C), por separado. Obtenidos los puntos que nos indican para cada $\mathrm{pH}$ el número de especies distintas de algas, buscamos un ajuste matemático que nos lo relacione, habiéndonos parecido la regresión lineal el mas apropiado.

De la pendiente positiva de la recta de regresión se deduce que, entre los márgenes de $\mathrm{pH}$ con los que trabajamos, al aumentar el $\mathrm{pH}$ aumenta la heterogeneidad algal (fig. 1 A). Con las cianofíceas es más acusada la relación (fig. 1 B) y el pH no tiene efecto sobre la heterogeneidad de las clorofíceas (fig. $1 \mathrm{C}$ ).

Para determinar la bondad del ajuste de las rectas hallamos el coeficiente de regresión, el cual según las tablas de Fisher y Yates (Schwartz, 1963)es fiable con una seguridad superior al $99 \%$.

El otro dato en estudio relacionado con la heterogeneidad algal ha sido la materia orgánica, de cuyo efecto sobre las algas del suelo no hemos encontrado reseñas bibliográficas. El porcentaje de M.O. sobre materia seca oscila entre 0 y 14 $\%$.

$\mathrm{Al}$ igual que con el $\mathrm{pH}$ hemos realizado gráficas, colocando los valores de porcentaje de materia orgánica en abscisas y en ordenadas la heterogeneidad algal (fig. $2 \mathrm{~A}$ ), el número de cianofíceas (fig. $2 \mathrm{~B}$ ) o el número de clorofíceas (fig. $2 \mathrm{C}$ ). En la figura número $2 \mathrm{~A}$ la pendiente de la recta es negativa y el coeficiente de regresión es de la misma fiabilidad que en el caso del $\mathrm{pH}$. El porcentaje de materia orgánica presente en el suelo tiene mayor efecto sobre el número total de especies algales 
(fig. 2A) y sobre las cianofíceas (fig. 2 B) que en las clorofíceas (fig. 2C), entre las que encontramos especies con heterotrofia facultativa.

Las gráficas nos revelan la gran influencia inhibitoria que la materia orgánica tiene sobre las algas edáficas. Sólo pueden resistir condiciones de elevada concentración de materia orgánica unas pocas especies calificadas como polisaprobias. Este hecho es ya ampliamente conocido en el medio acuático.

\section{BIBLIOGRAFIA}

AKIYAMA, 1973. Ecological studies on terrestrial algae found in the coastal sand dune in San-in region, Japan. Memoirs of the Faculty of Education Shimane University. 7 -Natural Science) Matsue. Japan.

BROCK, T., 1973. Lower pH limit for the existence of Blue-Green Algae: Evolutionary and Ecological Implications. Science. 179: 480-483.

HALPERIN, D. R., 1976. Biodermas algales como fuente de nitrógeno en suelos subhúmedos y semi-áridos. Darwiniana, 20 (3/4): 341-370.

LUND, J.W. G., 1947. Observations on soil algae II. Notes on groups other tham diatoms. New Phytol. 46: 35-60.

PANDEY, D. Ch., 1965. A Study on the algae from Paddy Field Soils of Ballia and Ghazipur districts of Uttar Pradesh. India. I. Cultural and ecological considerations. Nova Hedwigia 9 (1/4): 299-334.

SCHWARTZ, D., 1963. Méthodes statistiques a l'usage des médecins et des biologistes. Flammarion. Paris.

SHTINA, E. A., 1959. Algae solorum caespitoso-podzolensium regionis kirovskensis. Komarova Akad. Nauk. S. S. S.R. II. Sporovye Rasteniya 12: 36-141.

1960. Zonality in the distribution of soil algae communities. 7 th Inter. Congress of Soil Science, Madison. Wisc., U. S. A. 3 (24): 630-634. 

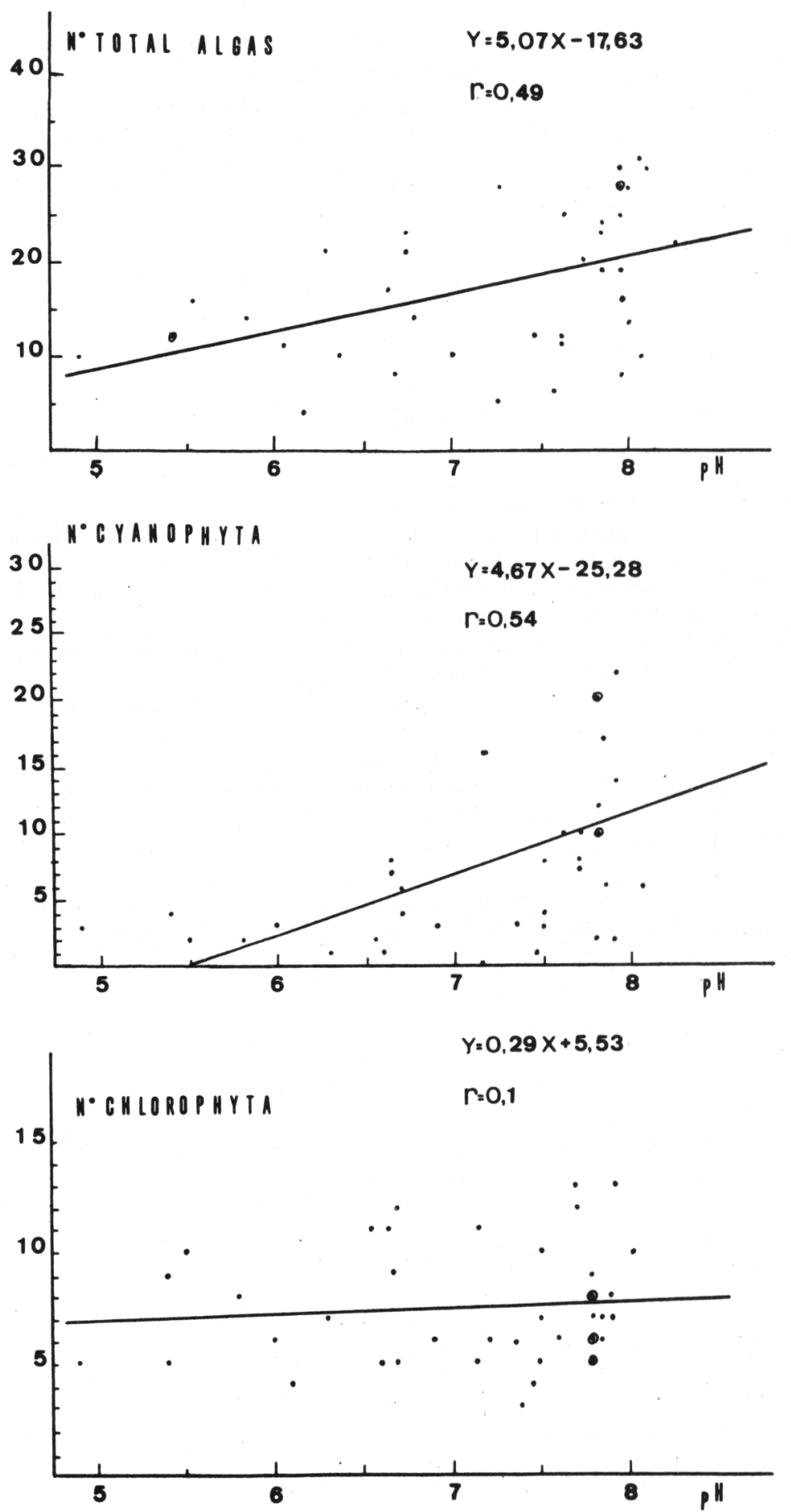

Figura 1.-Heterogenerdad algal en función del $\mathrm{pH}$. A, número total de especies; $\mathrm{B}$, Cyanophyta; C, Chlorophyta. 

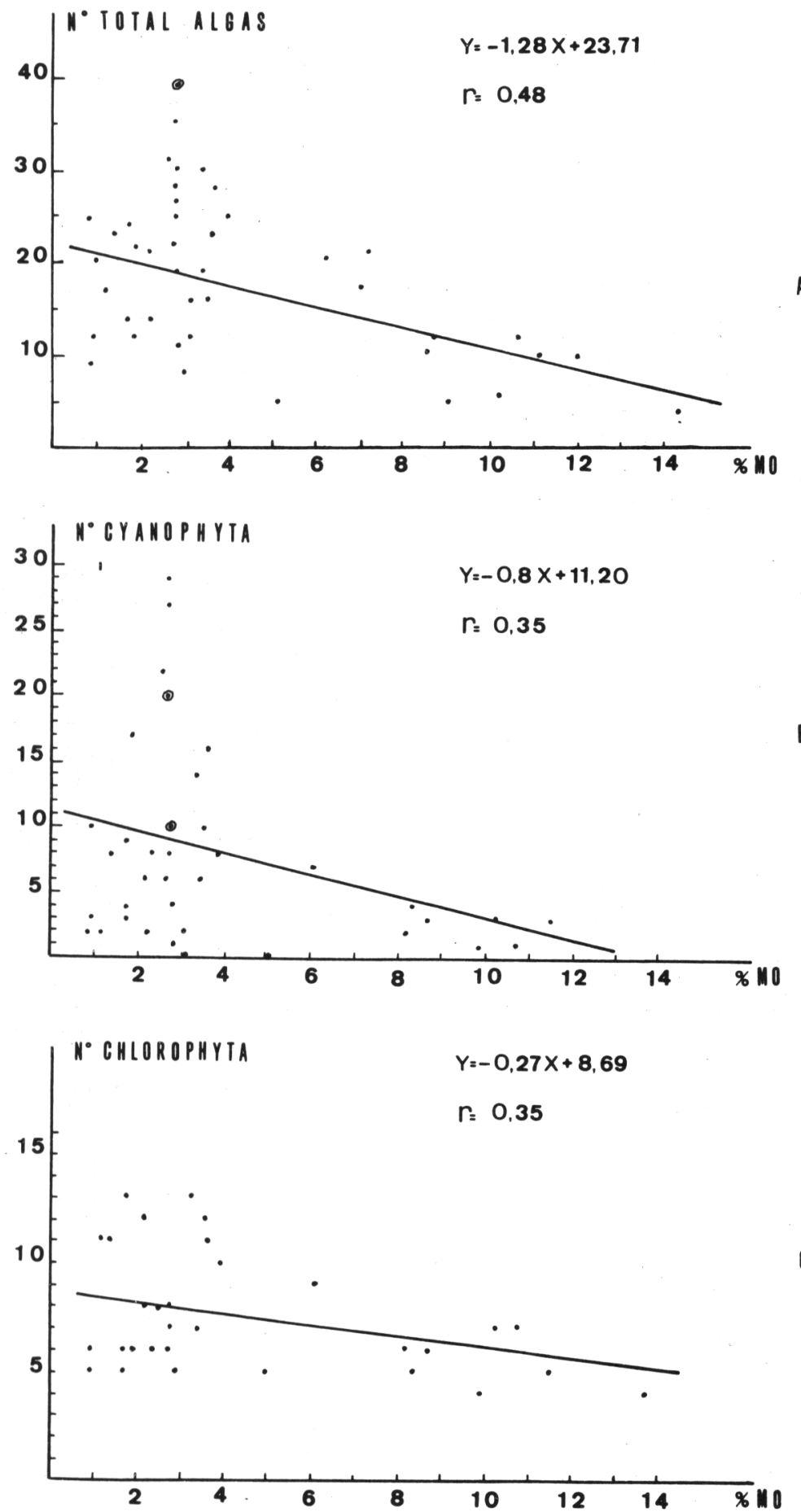

Figura 2.-Heterogeneidad algal en función de la materia orgánica A, Número total de especies; B, Cyanophyta; C, Chlorophyta. 
